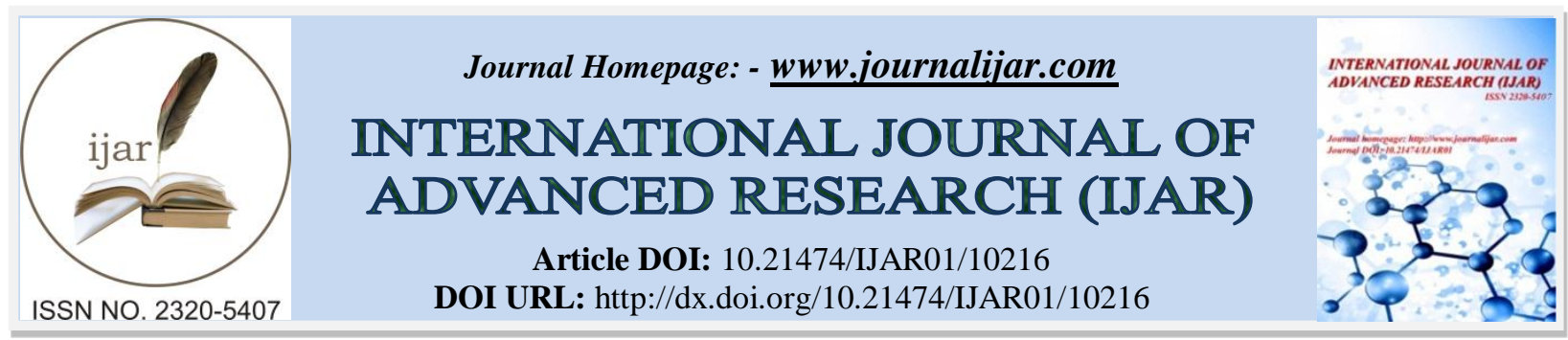

RESEARCH ARTICLE

\title{
FACTEURS PREDICTIFS D'UNE BONNE REPONSE AU TRAITEMENT PAR L'HORMONE DE CROISSANCE CHEZ LES ENFANTS AVEC RETARD STATURAL
}

\author{
M. Elbahi, G. Elmghari and N. Elansari \\ Service de Diabétologie, endocrinologie, maladies métaboliques et nutrition, Hôpital Arrazi, CHU Mohammed VI, \\ Université Cadi Ayad, Marrakech.
}

\section{Manuscript Info}

Manuscript History

Received: 10 October 2019

Final Accepted: 12 November 2019

Published: December 2019

\begin{abstract}
Object: Déterminer les facteurs prédictifs d'une bonne réponse thérapeutique chez les enfants sous hormone de croissance.

Matériel et Méthodes : Sont inclus dans cette étude les enfants sous GH pour déficit en hormone de croissance isolé ou dans le cadre d'une insuffisance hypophysaire multiple, un syndrome de Turner, un retard de croissance intra-utérin et une insuffisance rénale chronique, une approche statistique longitudinale a été utilisée, nous avons calculé le changement de la taille de référence en score de déviation standard ( $\triangle$ TSDS) après2ans de traitement en fonction des données cliniques (âge, sexe, IMC,), paracliniques (âge osseux, IGF1, pic de GH après stimulation), thérapeutiques (modalités du traitement par $\mathrm{GH}$ ) et évolutives (VC vitesse de croissance).

Résultats : Des augmentations graduelles du $\triangle$ TSDS au fil du temps ont été observées pour toutes les catégories diagnostiques. L'âge basal et l'âge osseux étaient négativement corrélés avec le $\triangle$ TSDS, la vitesse de croissance (VC) à 4mois, la taille initiale en score de déviation standard (SDS), l'IMC de base en (SDS) étaient positivement corrélées au $\triangle$ TSDS. L'IGF1en (SDS) et le sexe n'étaient pas statistiquement significatifs

Conclusion: Ces résultats peuvent aider à sélectionner les patients présumés meilleurs répondeurs au traitement tout en se basant sur leurs caractéristiques en prétraitement notamment; l'âge de référence, le TSDS, l'IMC(SDS), et leur vitesse de croissance à 3 mois.
\end{abstract}

Copy Right, IJAR, 2019,. All rights reserved.

\section{Introduction:-}

Le traitement par l'hormone de croissance exogène $(\mathrm{GH})$ est devenu une option thérapeutique bien acceptée chez les enfants avec retard de croissance. Il a été démontré qu'il augmente à la fois la croissance à court terme et la taille adulte [1]. Toutefois, une variabilité de réponse à ce traitement a été signalée selon les différentes catégories diagnostiques.

Cette variabilité rend difficile la décision de traiter par l'hormone de croissance par rapport au moment adéquat pour le début du traitement et la posologie à utiliser [2].

\section{Corresponding Author:- M. Elbahi}

Address:- Service de Diabétologie, endocrinologie, maladies métaboliques et nutrition, Hôpital Arrazi,

CHU Mohammed VI, Université Cadi Ayad, Marrakech. 
Les rapports d'essais cliniques suggèrent de nombreux facteurs qui influencent la réponse au traitement par $\mathrm{GH}$, telle que la réponse de croissance en première année, le plus jeune âge pour débuter le traitement, la différence de taille au début du traitement par rapport au score SD (TSDS), et la dose de GH [3,4]. Ces facteurs ont le potentiel d'aider à la planification individualisée du traitement par l'hormone de croissance et à l'adaptation du traitement basée sur les réponses précoces.

L'objectif de notre travail est de déterminer les facteurs prédictifs d'une bonne réponse thérapeutique chez les enfants sous hormone de croissance.

\section{Matériel et Méthodes:-}

Sont inclus dans cette étude les enfants sous GH pour: déficit en hormone de croissance isolé ou dans le cadre d'une insuffisance hypophysaire multiple, un syndrome de Turner, un retard de croissance intra-utérin et une insuffisance rénale chronique.

Les facteurs prédictifs ont été identifiés chez les patients avec déficit en GH à partir du changement de taille de référence en score de déviation standard ( $\Delta$ TSDS) après 24 mois de traitement.

Les données recueillies lors de la première consultation et / ou au cours du suivi étaient comme suivant : L'âge, le sexe, la dose de GH ( $\mu \mathrm{g} / \mathrm{Kg} / \mathrm{J})$, la TSDS (taille en score de déviation standard), l'insuline growth factor (IGF-I) SDS, l'indice de masse corporelle (IMC) SDS, l'âge osseux(AO), la vitesse de croissance(VC) annuelle, La concentration sérique maximale de $\mathrm{GH}$ stimulée $(\mathrm{ng} / \mathrm{ml})$.

La taille et l'IMC en SDS ( $Z$ score) ont été calculés selon la formule standard fournie par le centre pour le contrôle et la prévention des maladies [5].

L'Insuline growth Factor (IGF-I) SDS était calculé en utilisant un calculateur en ligne du service d'endocrinologie et des maladies de reproduction CHU Paris-Sud hôpital Bicêtre.

Les données ont été recueillies avant l'instauration du traitement, à 4 mois à un 1 an et à 24 mois du traitement par $\mathrm{GH}$.

La saisie informatique et le traitement des données ont été réalisés à l'aide des logiciels informatiques (EXCEL 2010 et IBM SPSS STATISTICS 20) et on a adopté des variables simples telles les fréquences, les pourcentages et les moyennes

\section{Résultats:-}

\section{I-Données démographiques de base:}

Nous avons recruté dans cette étude 20 patients avec retard staturo-pondéral dont 9 avec déficit antéhypophysaire multiple(DAM) ,4 avec déficit en GH (DGH) isolé, 2 avec insuffisance rénale chronique (IRC), 3 avec retard de croissance intra-utérin (RCIU)et 2 patientes avec un syndrome de Turner(ST).

Les données dermographiques de base sont résumées dans le (tableau 10). Les âges moyens initiaux étaient plus bas pour les groupes DAM (10,8 ans), RCIU (10,8 ans) par rapport au ST (12,9 ans), DGH (13ans) et IRC(14ans).

Les valeurs maximales moyennes de base de la GH étaient les plus faibles chez les patients atteints de DAM $(2,4 \mathrm{ng} / \mathrm{ml})$; les tests de stimulation de GH n'étaient pas nécessaire en cas ST, RCIU et IRC.

La dose de GH moyenne de base ( $\mu \mathrm{g} / \mathrm{kg} /$ jour) pour les différentes catégories de diagnostic était la plus faible pour les patients atteints de DAM, DGH et de RCIU, Pour toutes les catégories diagnostiques, les doses de GH moyenne et médiane n'ont pas augmenté de plus de $0,01 \mathrm{mg} / \mathrm{kg} /$ jour au cours des deux années, ce qui indique un changement très étroit de la dose de GH au cours de cette période. 
Tableau 1:- Données démographiques de référence par catégorie diagnostique.

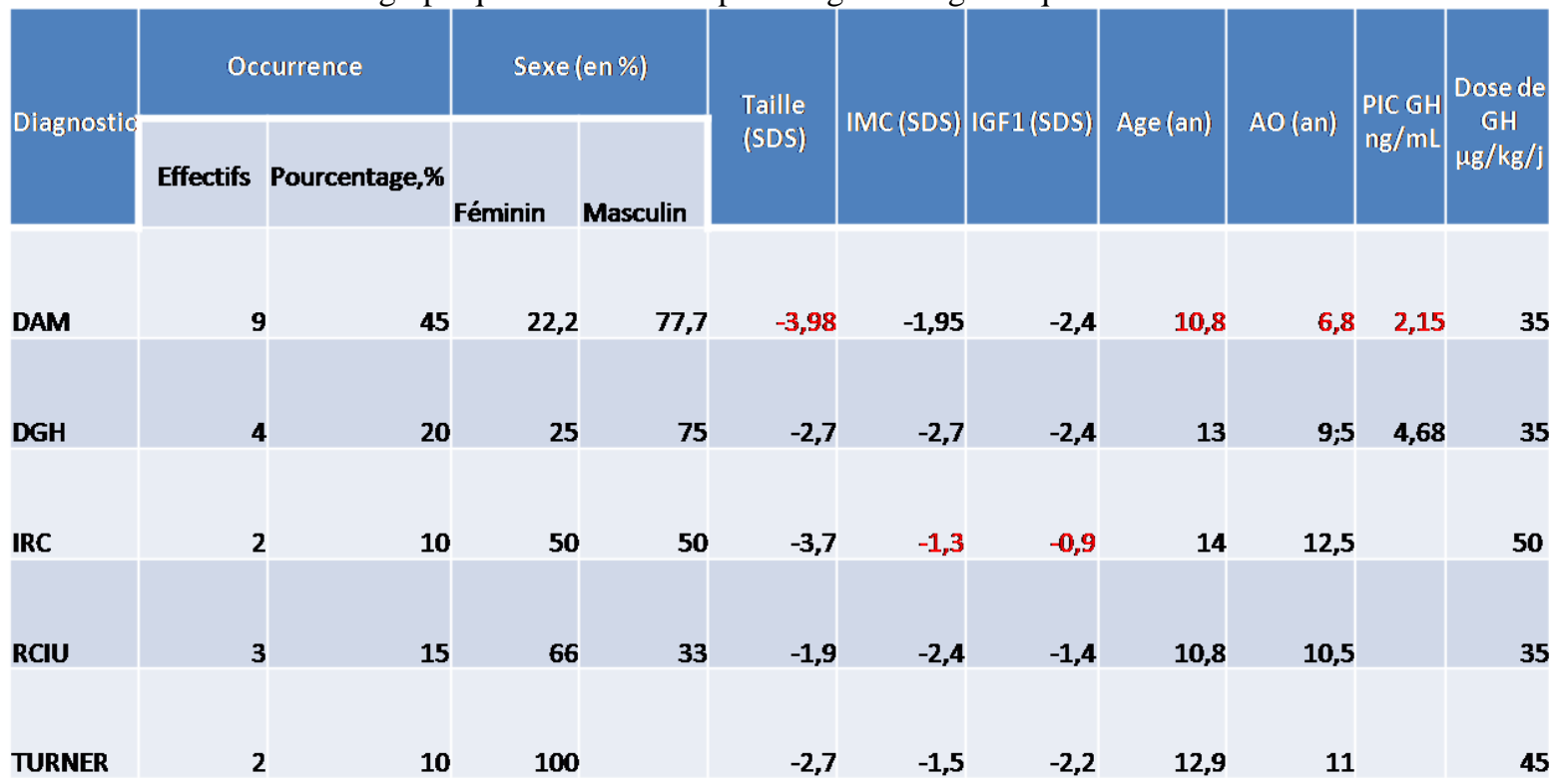

Résultats sur la taille:-

Le $\triangle$ TSDS:

Des augmentations graduelles du $\triangle \mathrm{TSDS}$ au fil du temps ont été observées pour toutes les catégories diagnostiques.

Elles variaient entre: 0,06 (IRC) à 0,34 (DAM) à 4 mois, de 0,26 (DGH) à 0,98 (DAM) à 12 mois, de 0,73 (ST) à 1,88

(DAM) à 24 mois.

Tableau 2:- Résultat sur le $\triangle T$ TSDS selon les catégories diagnostiques.

\begin{tabular}{|c|c|c|c|c|c|c|c|c|c|c|c|c|c|c|c|}
\hline & \multicolumn{3}{|c|}{ DAM } & \multicolumn{3}{|c|}{ DGH } & \multicolumn{3}{|c|}{ RCIU } & \multicolumn{3}{|c|}{ IRC } & \multicolumn{3}{|c|}{ TURNER } \\
\hline & \multicolumn{2}{|c|}{ TSDS } & \multirow{2}{*}{$\begin{array}{l}\text { ATSDS } \\
\mathbf{m}\end{array}$} & \multicolumn{2}{|c|}{ TSDS } & \multirow{2}{*}{$\begin{array}{l}\text { ATSDS } \\
\mathbf{m}\end{array}$} & \multicolumn{2}{|c|}{ TSDS } & \multirow{2}{*}{$\begin{array}{l}\text { ATSDS } \\
\mathbf{m}\end{array}$} & \multicolumn{2}{|c|}{ TSDS } & \multirow{2}{*}{$\begin{array}{l}\text { ATSDS } \\
\mathbf{m}\end{array}$} & \multicolumn{2}{|c|}{ TSDS } & \multirow{2}{*}{$\begin{array}{l}\Delta T S D S \\
\text { m }\end{array}$} \\
\hline & $\mathbf{n}$ & m & & $\mathbf{n}$ & m & & $\mathbf{n}$ & m & & $\mathbf{n}$ & m & & $\mathbf{n}$ & m & \\
\hline $\begin{array}{l}\text { Age } \\
\text { basal }\end{array}$ & 9 & 4,47 & - & 4 & $-3,07$ & - & 3 & 1,29 & - & 2 & $-3,65$ & - & 2 & $-4,27$ & - \\
\hline 4 mois & 8 & 3,98 & 0,34 & 4 & $-2,51$ & 0,16 & 3 & 1,42 & 0,5 & 2 & $-3,59$ & 0,06 & 2 & $-4,02$ & 0,25 \\
\hline 18 mois & 9 & 3,46 & 0,98 & 4 & $-2,03$ & 0,26 & 2 & 1,36 & 0,93 & 2 & $-3,22$ & 0,43 & 2 & $-3,87$ & 0,4 \\
\hline 24 mois & 8 & 3,37 & $1 ; 88$ & 3 & $-1,77$ & 0,85 & 2 & 1,22 & 1,07 & 2 & $-2,89$ & 0,76 & 2 & $-3,54$ & 0,73 \\
\hline
\end{tabular}

La vitesse de croissance :

Selon chaque catégorie diagnostique, la vitesse de croissance annuelle augmentait au fil des mois du traitement. La VC moyenne annuelle à 1 an était plus élevée pour le RCIU à 10,43cm/ an, et entre 9 (TS, IRC) et 10,27 cm/an (DAM) pour les autres catégories diagnostiques.

Dans chaque catégorie de diagnostic, la VC annuelle moyenne était plus élevée au cours de la première année et a généralement diminué au cours de la deuxième année. La VC annuelle moyenne à 1 an était la plus élevée pour le DAM à 10,64 cm / an et variait entre 7,73 (ST) et 9,66 (DGH) pour les autres indications. 
Figure 1:- Vitesse de croissance selon les catégories diagnostiques.

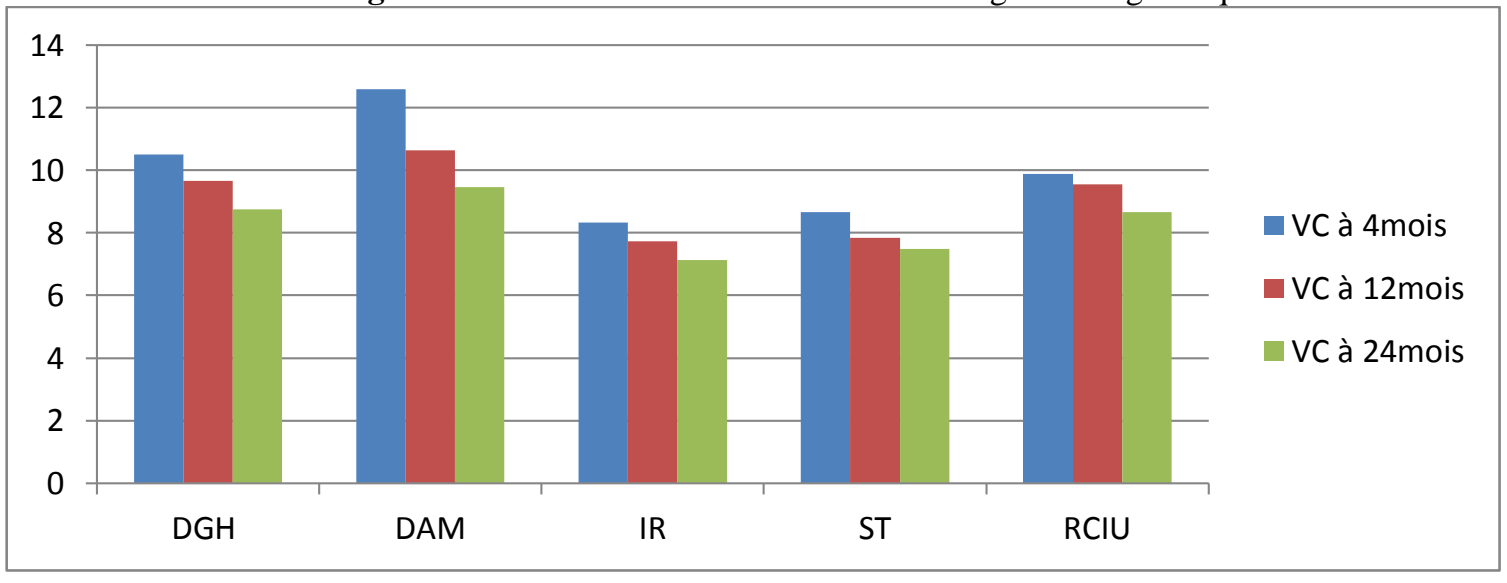

Résultats des corrélations du $\triangle T$ TSDS et autres paramètres:

L'âge basal, la taille initiale en score de déviation standard (SDS) et l'âge osseux étaient négativement corrélés avec le $\triangle$ TSDS, la vitesse de croissance (VC) à 4mois, l'IMC de base en (SDS) étaient positivement corrélées au $\Delta$ TSDS. L'IGF1en (SDS) et le sexe n'étaient pas statistiquement significatifs dans cette étude.

Tableau 2:- Corrélation du $\triangle \mathrm{TSDS}$ avec VC à 4mois et d'autres paramètres dermographique (corrélation de Pearson).

\begin{tabular}{|l|l|l|l|l|l|l|l|} 
& Sexe & Age & $\begin{array}{l}\text { IMC } \\
\text { (SDS) }\end{array}$ & $\begin{array}{l}\text { IGF1 } \\
\text { (SDS) }\end{array}$ & $\begin{array}{l}\text { Taille } \\
\text { (SDS) }\end{array}$ & $\begin{array}{l}\text { A0 } \\
\text { (ans) }\end{array}$ & $\begin{array}{l}\text { VCà } \\
4 \text { mois }\end{array}$ \\
\hline $\begin{array}{l}\Delta T S D S \\
12 \text { mois }\end{array}$ & 0,772 & $-0,072$ & 0,032 & $-0,389$ & $-0,058$ & $-0,059$ & 0,070 \\
\hline $\begin{array}{l}\Delta T S D S \\
24 \text { mois }\end{array}$ & 0,895 & $-0,010$ & 0,042 & $-0,517$ & $-0,0316$ & $-0,017$ & 0,089 \\
\hline
\end{tabular}

\section{Discussion:-}

À travers cette étude longitudinal; plusieurs variables identifiés ont été corrélés avec la réponse de la croissance pendant la première année et la $2^{\text {ème }}$ année du traitement par la GH. La vitesse de croissance à 4 mois était positivement corrélée au $\triangle \mathrm{TSDS}$ : Cette observation est une découverte novatrice, étant donné que de nombreuses études ne signalent pas leur croissance au début du cycle de traitement. L'âge basal, la taille de référence et l'IMC avaient également une influence significative sur la prédiction de la réponse à l'hormone de croissance.

Les résultats de cette étude concordent avec les résultats d'études antérieurs menées chez des patients présentant un retard staturo-pondéral en rapport avec des pathologies spécifiques : En cas d'un déficit en GH lee and al retrouvaient que la $\mathrm{VC}$ à 4 mois et l'IMC de base en SDS étaient positivement corrélées au $\Delta T$ TSDS, l'âge de référence, la Taille et l'IGF-I de référence en SDS y étaient négativement corrélée [6].

Cependant certaines études ont rapporté d'autres facteurs significatifs sur la réponse à la GH: en cas de déficit en GH Selon Ranke et al Le poids à la naissance en SDS était corrélé avec la réponse de croissance à la GH [7], suggérant que plus le poids de naissance était élevé, plus la réponse au traitement par la GH était importante. En cas d'un syndrome de Turner traité pendant une longue durée (2-4ans): la dose de GH par semaine, le poids en SDS, l'âge chronologique, la vitesse de croissance durant les années antérieures du traitement, le traitement adjuvant à l'oxandrolone Sont considérés comme des facteurs prédictifs significatifs influençant la réponse à la GH [8]. En cas 
de RCIU : la réponse de la croissance après un 1 an de traitement par GH était le facteur prédictif le plus important pour une bonne réponse après la $2^{\text {ème }}$ année du traitement [9]

Les résultats spécifiques d'autres études qui sont cohérents avec la présente analyse, incluent le manque de l'effet du sexe sur la réponse au traitement par la GH[10].L' analyse des résultats de l'étude internationale sur la croissance de Pfizer Kabi a montré qu'il n'existe aucune différence significative entre les sexes dans les effets de la GH sur la vitesse de croissance ou TSDS sur 2 ou 3 ans de traitement [10].

Dans un rapport récent, une grande cohorte de patients masculins et féminins atteints de DAM, ST, RCIU, syndrome de noonan et retard de croissance idiopathique du registre ANSWER (American Norditropin Studies: Web-enabled Research) a été utilisé pour évaluer les différences dans $\triangle$ TSDS liées au genre après 2 ans de traitement par GH. Les résultats ont montré une augmentation du $\triangle$ HSDS chez tous les patients, cependant, les différences cliniquement pertinentes entre les sexes n'étaient pas observées [11].

Les différentes corrélations entre l'âge de référence, TSDS, IMC en SDS, IGF-I en SDS, et la vitesse de croissance à 3 mois avec la réponse de croissance sur 24 mois de traitement par la $\mathrm{GH}$, ont d'énormes répercussions pratiques.

La corrélation négative de l'âge basal avec $\triangle$ TSDS chez nos patients soutient l'initiation de la GH à un âge aussi jeune que possible pour favoriser une croissance optimale.

Ce concept est supporté par les résultats d'une autre étude qui a démontré une relation entre l'âge de référence et la VC de première année chez les patients atteints de DGH, DAM et ST [12]. Bien que la proportion de patients pubères dans la présente analyse soit indéfinie, la moyenne de l'âge osseux est compatible avec la probabilité que la majorité de nos patients sont non pubères, ce qui réduit l'impact de la puberté sur la réponse à la GH.

Une corrélation négative a été observée entre l'IGF-I de base et le changement de TSDS sur deux ans de traitement par GH ce qui est concordant avec une sensibilité accrue aux effets de la GH chez les patients avec un déficit en GH plus en moins sévère. Dans cette

étude, le dosage d' IGF-I a été mesurée dans un certain nombre de laboratoires commerciaux reflétant la pratique clinique de routine. L'IGF-I en SDS a été calculée en utilisant une formule qui a fourni une cohérence à l'analyse. Cette coherence qui ce reflète également dans la conclusion de nos résultats selon lesquelles l' IGF-I en SDS de base moyen chez les patients avec DGH et DAM était inférieure à celui observé chez les non-déficitaires en GH. La corrélation positive entre l'IMC de base en SDS et le $\triangle$ TSDS dans cette étude pourrait refléter, du moins en partie, l'importance de la nutrition pour l'optimisation des résultats chez les patients recevant GH [13].Un IMC anormalement bas chez les enfants peut être un signe de malnutrition, qui peut également être associé à une anomalie de croissance.

\section{Conclusion:-}

Les résultats de cette étude peuvent aider à guider le traitement par GH par une sélection des patients présumés meilleurs répondeurs au traitement; Une sélection basée sur leurs caractéristiques en prétraitement notamment ; l'âge de référence, la Taille initiale en SDS, l'IMC en SDS, et la vitesse de croissance à 4 mois. le sexe reste un facteur non significatif sur la réponse à l'hormone de croissance.

\section{Reference:-}

1. Sas TC, de Ridder MA, Wit JM, Rotteveel J, Oostdijk W, Reeser HM, Otten BJ, de Muinck Keizer-Schrama SM: Adult height in children with growth hormone deficiency: a randomized, controlled, growth hormone doseresponse

2. trial. Horm Res Paediatr 2010, 74:172-181.

3. Hintz RL: Growth hormone treatment of idiopathic short stature: clinical studies. Growth Horm IGF Res 2005, 15(suppl A):S6-S8.

4. Zucchini S: Growth hormone use in the treatment of idiopathic short stature. Curr Opin Invest Drugs 2008, 9:396-401. 
5. Ranke MB, Lindberg A, Price DA, Darendeliler F, bertsson-Wikland K, Wilton P, Reiter EO: Age at growth hormone therapy start and first-year responsiveness to growth hormone are major determinants of height outcome in idiopathic short stature. Horm Res 2007, 68:53-62.

6. US Centers for Disease Control and Prevention: Percentile data files with LMS values.

7. Lee, P. A., Germak, J., Gut, R., Khutoryansky, N., \& Ross, J. (2011). Identification of factors associated with good response to growth hormone therapy in children with short stature: results from the ANSWER Program®. International journal of pediatric endocrinology, 2011(1), 6.

8. Ranke MB, Lindberg A, Chatelain P, Wilton P, Cutfield W, bertsson-Wikland K, Price DA: Derivation and validation of a mathematical model for predicting the response to exogenous recombinant human growt hormone $(\mathrm{GH})$ in prepubertal children with idiopathic GH deficiency. KIGS International Board. Kabi Pharmacia International Growth Study. J Clin Endocrinol Metab 1999, 84:1174-1183.

9. Ranke MB, Lindberg A, Chatelain P, Wilton P, Cutfield W, bertsson-Wikland K, Price DA: Prediction of longterm response to recombinant human growth hormone in Turner syndrome: development and validation of mathematical models. KIGS International Board. Kabi International Growth Study. J Clin Endocrinol Metab 2000, 85:4212-4218.

10. Ranke MB, Lindberg A, Cowell CT, Wikland KA, Reiter EO, Wilton P, Price DA:Prediction of response to growth hormone treatment in short children born small for gestational age: analysis of data from KIGS (Pharmacia International Growth Database). J Clin Endocrinol Metab 2003, 88:125-131.

11. Rose SR, Shulman DI, Larsson P, Wakley LR, Wills S, Bakker B: Gender does not influence prepubertal growth velocity during standard growth hormone therapy-analysis of United States KIGS data. J Pediatr Endocrinol

12. Metab 2005, 18:1045-1051.

13. Ross J, Lee PA, Gut R, Germak J: Factors influencing the one- and twoyear growth response in children treated with growth hormone: analysis from an observational study. Int J Pediatr Endocrinol 2010, 2010:494656.

14. Bakker B, Frane J, Anhalt H, Lippe B, Rosenfeld RG: Height velocity targets from the national cooperative growth study for first-year growth hormone responses in short children. J Clin Endocrinol Metab 2008, 93:352357.

15. Grumbach MM, Bin-Abbas BS, Kaplan SL: The growth hormone cascade: progress and long-term results of growth hormone treatment in growth hormone deficiency. Horm Res 1998, 49(suppl 2):41-57. 